\title{
High-Peak Power Frequency Modulation Pulse Generation in Cascaded Fiber Configurations with Inscribed Fiber Bragg Grating Arrays
}

\author{
Aleksei Abramov ${ }^{1}$, Igor Zolotovskii ${ }^{1, *}$, Vladimir Kamynin ${ }^{2}$, Victor Prikhodko ${ }^{1}$, Aleksei Tregubov ${ }^{1}$, \\ Dmitrii Stoliarov $^{1}{ }^{1}$, Marina Yavtushenko ${ }^{1}$ and Andrei Fotiadi ${ }^{1,3}{ }^{\mathbb{D}}$ \\ 1 S.P. Kapitsa Scientific Technological Research Institute, Ulyanovsk State University, 42 Leo Tolstoy Street, \\ 432970 Ulyanovsk, Russia; aleksei_abramov@mail.ru (A.A.); v_prikhodko@mail.ru (V.P.); \\ tregub@ulsu.ru (A.T.); dmitreyst@gmail.com (D.S.); myavtushenko@mail.ru (M.Y.); fotiadi@mail.ru (A.F.) \\ 2 Prokhorov General Physics Institute of the Russian Academy of Sciences, 38 Vavilov Str., \\ 119991 Moscow, Russia; kamyninva@gmail.com \\ 3 Electromagnetism and Telecommunication Department, University of Mons, B-7000 Mons, Belgium \\ * Correspondence: rafzol.14@mail.ru
}

Citation: Abramov, A.; Zolotovskii,

I.; Kamynin, V.; Prikhodko, V.;

Tregubov, A.; Stoliarov, D.;

Yavtushenko, M.; Fotiadi, A.

High-Peak Power Frequency

Modulation Pulse Generation in

Cascaded Fiber Configurations with

Inscribed Fiber Bragg Grating Arrays.

Photonics 2021, 8, 471. https://

doi.org/10.3390/photonics 8110471

Received: 18 September 2021

Accepted: 21 October 2021

Published: 24 October 2021

Publisher's Note: MDPI stays neutral with regard to jurisdictional claims in published maps and institutional affiliations.

Copyright: (C) 2021 by the authors Licensee MDPI, Basel, Switzerland. This article is an open access article distributed under the terms and conditions of the Creative Commons Attribution (CC BY) license (https:// creativecommons.org/licenses/by/ $4.0 /)$

\begin{abstract}
We explored the dynamics of frequency-modulated (FM) pulses in a cascaded fiber configuration comprising one active and one passive optical fiber with multiple fiber Bragg gratings (FBGs) of different periods inscribed over the fiber configuration length. We present a theoretical formalism to describe the mechanisms of the FM pulse amplification and pulse compression in such fiber cascades resulting in peak powers up to $\sim 0.7 \mathrm{MW}$. In combination with the decreasing dispersion fibers, the considered cascade configuration enables pico- and sub-picosecond pulse trains with a sub-terahertz repetition rate and sub-kW peak power generated directly from the continuous optical signal.
\end{abstract}

Keywords: generation of ultrashort pulses; fiber Bragg gratings; frequency-modulated pulse temporal compression; modulation instability

\section{Introduction}

Fiber laser technology has recently been improved with the emerging ultrashort pulse (USP) sources emitting pulses of high peak power. Such laser sources are in great demand for a variety of applications, e.g., material processing, cut edging, precise surface structuring, and surgical procedures. In science and metrology, USP lasers are used with ultrafast spectroscopy, multiphoton microscopy, or optical coherence tomography [1-4]. The achievement of higher pulse peak power in fiber lasers is associated with the use of optical fibers possessing a low nonlinearity [5,6]. Among such fiber materials are singlemode fibers with large mode areas $\left(>100 \mu \mathrm{m}^{2}\right)$ [5-10] and step- or grade-index tapered fibers [8-14]. Additionally, the optical fibers with the dispersion varying along the fiber length are commonly used for the generation of high peak power USPs [1-6]. Such fibers also enable pulse compression, supercontinuum generation, and optical processing $[15,16]$.

Therefore, the design of nonuniform fibers possessing low nonlinearity is of great practical interest for high-power USP fiber lasers. However, the precise control of the fiber dispersion parameters is a technically challenging task. In particular, in gradeindex (parabolic) and step-index tapered fibers, the group velocity dispersion (GVD) is determined by the fiber material dispersion and cannot be tailored. A simple way to overcome this limitation is to inscribe the array of refractive index fiber Bragg gratings with different periods distributed along the fiber length in a proper way. This concept has been demonstrated to control the frequency-modulated (FM) pulse dynamics in [17-21].

Currently, FBG fabrication is a well-established technique [22,23]; in particular, it is widely used with grade-index multimode fibers and is promising for applications in high- 
energy and high-peak power laser pulsed sources [24-30]. At present, FBGs are widely used both as elements of various types of fiber lasers and as sensitive elements of various fiber sensors [31-38]. Now, there are many papers in which the spectral characteristics of FBGs, their manufacturing methods, and their application possibilities are considered.

In this paper, we describe the method enabling amplification and temporal compression of FM pulses in a fiber configuration comprising both passive and active (amplifying) fibers with inscribed refractive index fiber Bragg gratings (FBGs) of different periods. We demonstrate that, in combination with the tapered fiber, such a fiber configuration could be used for the generation of an ultrashort pulse train with a high repetition rate (terahertz) directly from the weakly modulated CW input light.

\section{Fibers with Inscribed FBG Arrays}

Let us consider the dynamics of an optical signal propagating in a fiber with inscribed FBG arrays (Figure 1). The refractive index variation along the FBG is defined by the function $[15-19,39,40]$ :

$$
n_{i}(z)=n_{0}\left[1-m \cos \left(2 \pi z / \Lambda_{i}(z)\right)\right]
$$

where $n_{0}$ is the fiber average refractive index, $m$ is the modulation depth of the inscribed grating, and $\Lambda_{i}$ is the period of the $i$-th FBG, $i=1,2 \ldots$

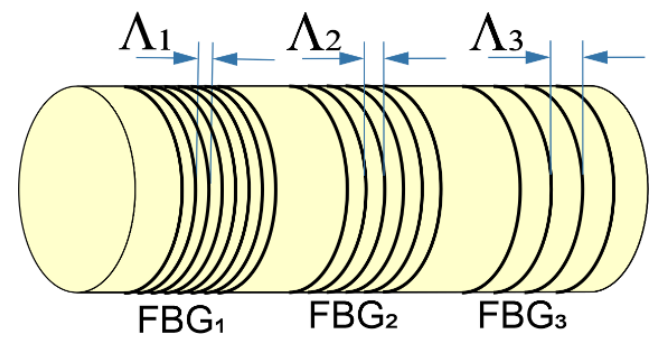

Figure 1. Optical fiber segment comprising three FBGs with increasing period.

The contribution of the refractive index changes in the FBG to the GVD is known to dominate the fiber material dispersion. The second-order GVD is expressed as $[15,16,28,31]$ :

$$
\beta_{2}(z)=d_{2}-\frac{\operatorname{sign}(\delta) \cdot \kappa^{2}}{V_{g}^{2}\left(\delta^{2}-\kappa^{2}\right)^{3 / 2}}=\frac{1}{c}\left(\frac{\partial^{2}\left(\omega n_{0}(\omega)\right)}{\partial \omega^{2}}\right)_{\omega=\omega_{0}}-\frac{\operatorname{sign}(\delta) \cdot \kappa^{2}}{V_{g}^{2}\left(\delta^{2}-\kappa^{2}\right)^{3 / 2}}
$$

where $d_{2}$ is the fiber material second-order dispersion, $V_{g}$ is the group velocity of the pulse propagating in the fiber without FBGs, $\kappa=2 \pi m / \lambda_{0}$ is the coefficient describing the coupling between the forward and backward waves, $\lambda_{0}$ is the central optical signal wavelength, and $\delta_{i}$ is the normalized detuning of the optical signal carrier frequency from the peak FBG reflectivity frequency $\omega_{B}$.

For a uniform FBG, this detuning can be written as [15,16,28,31]:

$$
\delta_{i}(z)=\frac{\omega_{0}-\omega_{B i}(z)}{V_{g}}=\frac{2 \pi c}{V_{g}}\left(\frac{1}{\lambda_{0}}-\frac{1}{\lambda_{B i}(z)}\right)=\frac{2 \pi c}{V_{g} \lambda_{0}}-\frac{\pi}{\Lambda_{i}(z)} .
$$

Depending on $\Lambda_{i}(z)$ the detuning $\delta_{i}(z)$ can be either negative or positive. Correspondingly, the second-order GVD of the fiber is normal or anomalous at $\delta<0$ or $\delta>0$, respectively.

Hereinafter, the pulse wavelength is assumed to be less than and far from the FBG peak reflectivity wavelength, i.e., $\delta \gg \kappa$. In this case, Equation (2) is reduced to:

$$
\beta_{2}(z)=d_{2}-\frac{\kappa^{2}}{V_{g}^{2} \delta^{3}}
$$

The FBG reflectivity spectrum bandwidth is $\Delta \lambda_{\text {gapp }}=m \lambda_{0} / 2 n_{0} \ll 1 \mathrm{~nm}$. 
At a large frequency detuning $\delta \gg \kappa$, the inscribed FBG has no effect on the effective cubic nonlinear coefficient in the fiber since it is determined by the fiber material only. However, the contributions of higher-order dispersion coefficients still have to be taken into account. In particular, the third-order GVD contribution is expressed as $[15,16,28,31]$ :

$$
\beta_{3}(z)=d_{3}+\frac{3 \delta \kappa^{2}}{V_{g}^{3}\left(\delta^{2}-\kappa^{2}\right)^{5 / 2}}=\frac{1}{c}\left(\frac{\partial^{3}\left(\omega n_{0}(\omega)\right)}{\partial \omega^{3}}\right)_{\omega=\omega_{0}}+\frac{3 \kappa^{2}}{V_{g}^{3} \delta^{4}},
$$

In accordance with Equation (5), the parameter $\beta_{3}(z)$ in a single-mode fiber is several orders of magnitude higher than the material third-order dispersion $d_{3}[15,16]$, so it can deteriorate the FM pulse compression quality, resulting in pulse shape distortion and even decay.

Let the period $\Lambda_{i}(z)$ of FBGs inscribed in the optical fiber increase along the fiber length $z$. In this case, $\delta$ also increases with $z$, leading to a decrease in the anomalous GVD absolute value. As follows from Equations (2) and (5), the second- and third-order dispersion parameters decrease as $\delta^{-3}$ and $\delta^{-4}$, respectively. The contributions of higherorder dispersion $\left(\beta_{n} \sim \delta^{-n}\right)$ reduce even faster, thereby preventing shape deformation for the generation of high-peak power subpicosecond pulses considered below.

\section{Soliton-Like FM Pulse Temporal Compression in Fibers with Inscribed FBGs}

The dynamics of pulse propagation in an optical fiber with inscribed FBG arrays (Figure 1) can be described by the nonlinear Schrödinger equation commonly used with nonuniform fibers [29,33-35] and taking into account the fiber nonlinearity, gain factor, and second- and third-order dispersion coefficients:

$$
\frac{\partial A}{\partial z}-i \frac{\beta_{2}(z)}{2} \frac{\partial^{2} A}{\partial \tau^{2}}-\frac{\beta_{3}(z)}{6} \frac{\partial^{3} A}{\partial \tau^{3}}+i R(z)\left(|A|^{2}-\tau_{R} \frac{\partial|A|^{2}}{\partial \tau}\right) A=g(z) A
$$

where $\tau=t-\int_{0}^{z} d \xi / u(\xi)$ is the time in the running reference frame, $u(z)$ is the pulse group velocity, $\beta_{2,3}(z)$ are the second- and third-order dispersion parameters, $R(z)$ is the cubic (Kerr) nonlinearity coefficient, $g(z)$ is the gain factor, and $\tau_{R}$ is the nonlinear (Raman) response time in the fiber medium.

At $\beta_{3}(z) \rightarrow 0$ and $\tau_{R} \rightarrow 0$, the pulse evolution described by Equation (6) could exhibit unlimited temporal compression, resulting in an unlimited increase in the peak amplitude $[29,33,34]$. To obtain this solution, the dispersion and nonlinear coefficient should be kept unchangeable along the fiber with anomalous GVD $\left(\beta_{2} R<0\right)$, and the gain factor distribution determined by the function $g(z)=g_{0} /\left(1-2 g_{0} z\right)$ should be provided. In this case, for the fiber length $2 g_{0} z<1$, the pulse evolution described by Equation (6) is expressed as:

$$
A(\tau, z)=\frac{A_{0}}{1-2 g_{0} z} \operatorname{sech}\left(\frac{\tau}{\tau_{s}}\right) \exp \left(i \frac{\alpha_{0} \tau^{2}-\Gamma z}{1-2 g_{0} z}\right) .
$$

Here, $A_{0}, \tau_{s}, \alpha_{0}$ are the input pulse amplitude, duration, and FM rate, respectively, $\Gamma=g_{0} / 2 \alpha_{0} \tau_{0}^{2}$.

A similar scenario of the FM soliton pulse evolution is also achievable in the optical fiber with a constant gain but with the GVD varying along the fiber length. To obtain this operation in the active fiber with a constant gain factor, the GVD profile has to be described as [41-44]:

$$
\beta_{2}(z) \rightarrow-\left|\beta_{20}\right| \exp \left[-\frac{\alpha_{0}\left|\beta_{20}\right|}{g_{0}}\left(\exp \left(2 g_{0} z\right)-1\right)+2 g_{0} z\right] .
$$

This results in the evolution of the pulse duration:

$$
\tau(z) \rightarrow \tau_{0} \exp \left[-\frac{\alpha_{0}\left|\beta_{20}\right|}{g_{0}}\left(\exp \left(2 g_{0} z\right)-1\right)\right]
$$


For passive fibers with $g_{0}=0$, Equations (8) and (9) are reduced to:

$$
\begin{gathered}
\beta_{2}(z) \approx-\left|\beta_{20}\right| \exp \left(-2 \alpha_{0}\left|\beta_{20}\right| z\right), \\
\tau_{s}(z) \approx \tau_{0} \exp \left(-2 \alpha_{0}\left|\beta_{20}\right| z\right),
\end{gathered}
$$

Therefore, under the condition that the proper GVD profile is provided by a proper distribution of the inscribed FBG period along the fiber length, fibers with inscribed FBG arrays could be employed for the generation of high-peak power USPs.

Let us consider the dynamics of an FM pulse propagating in a passive or active optical fiber with an inscribed FBG array (Figure 1). All inscribed FBGs differ only in the period $\Lambda_{i}$, where $i$ is the FBG order number. The average refractive index $n_{0}=1.5$ and modulation depth $m$ are assumed to be the same for all FBGs.

In the numerical calculations, we used the parameters typical for standard optical fibers and inscribed gratings: the cubic nonlinearity coefficient $R=10^{-3}(\mathrm{~W} \times \mathrm{m})^{-1}$ at the wavelength $\lambda_{0}=1550 \mathrm{~nm}$, the mode effective area $S_{\text {eff }}=50 \mu \mathrm{m}^{2}$, and the nonlinear response time of the medium of $\tau_{R} \approx 5 \times 10^{-15} \mathrm{~s}, d_{2}=-2 \times 10^{-27} \mathrm{~s}^{2} / \mathrm{m}, d_{3}=10^{-40} \mathrm{~s}^{3} / \mathrm{m}[15,16]$. It is worth noting that the parameter $d_{2}$ can play a crucial role at the final stage of pulse compression when the light bandwidth is maximal and the effect of the inscribed FBG on the dispersion is minimal. The parameters used for the inscribed FBGs were the Bragg wavelength $\lambda_{B 1}(z=0) \equiv 2 n_{0} \Lambda_{1}=1556.5 \mathrm{~nm}$, detuning $\delta \approx 2.5 \times 10^{4} \mathrm{~m}^{-1}$, and refractive index modulation depth $m=5 \times 10^{-4}$. The coupling coefficient $\kappa \approx 2.5 \times 10^{3} \mathrm{~m}^{-1}$ corresponds to these parameters. The proper distribution of the FBG period along the fiber length was used to provide the exponential GVD profile $\beta_{2}(z)=-\left|\beta_{20}\right| \exp (-q z)$.

The optimal fiber parameters are those that enable the FM soliton pulse generation determined by relations (7) and (8) $[43,44]$. The generation of high-peak power FM pulses is provided by tailoring the GVD profile $\beta_{2}(z)$ and the proper selection of the initial frequency modulation rate $\alpha_{0}$. In turn, the $\beta_{2}(z)$ parameter is governed mainly by the variation of the FBG parameters according to (2) and (4), whereas higher-order dispersion has to be relatively low:

$$
\left|\beta_{2}\right|>>\left|\frac{\beta_{n} \Delta \omega^{n-2}}{n !}\right|, n \geq 3,
$$

where $\Delta \omega$ is the FM pulse spectrum width.

Figure 2 compares the results of the FM pulse (6) temporal compression in a $20 \mathrm{~cm}$ passive optical fiber comprising 5, 10, or 20 inscribed FBGs separated by 4, 2, or $1 \mathrm{~cm}$, respectively. The pulse duration at the fiber input is $\tau_{0}=10 \mathrm{ps}$, the FM rate is $\alpha_{0} \simeq 10^{23} \mathrm{~s}^{-2}$, and the peak power is $P_{0}=\left|A_{0}\right|^{2}=7.7 \mathrm{~kW}$. The output pulses obtained in three cases are shown in comparison with the similar pulse obtained in the fiber with the perfect exponential GVD profile. Along the fiber length, the GVD decreases from $\beta_{2}(0) \approx-5 \times 10^{-23} \mathrm{~s}^{2} / \mathrm{m}$ to $\beta_{2} \approx-2.5 \times 10^{-26} \mathrm{~s}^{2} / \mathrm{m}$, with the period increasing from 516 to $534 \mathrm{~nm}$. One can see that the discretization of the GVD profile into individual gratings leads to worse compression and causes an increase in the noise pedestal level. Nevertheless, the use of the fiber with at most 5 FBGs enables an effective compression of the initial pulse. A larger number of FBGs inscribed within the same fiber length provides a higher peak power and a smoother pedestal. We observed that the use of a fiber length larger than $20 \mathrm{~cm}$ causes rapid distortion of the FM pulse independently of the number of FBGs used. In this case, the less smooth the GVD profile is, the sooner the destruction occurs.

In our opinion, to implement the proposed scheme, the long chirped FBGs with a smooth change in the period seem to be the most suitable. However, as mentioned in our work, the inscription of the chirped FBGs is technically difficult. Therefore, the use of multiple uniform FBGs of different periods inscribed over the fiber length is a simpler way to provide a smooth decrease in the anomalous GVD induced by the FBG. Therefore, in the revised manuscript, Figure 2 shows the FM pulse peak power achieved in the fiber with a different number of FBGs. One can see that a chirped grating with a 
smooth change of the period ensures the most compression efficiency. On the other hand, discrete uniform gratings can also provide a good result. Moreover, the shorter the grating with a fixed period we used, the smoother dispersion profile that can be achieved. The analysis shows that an increase in the FBG number (within the same fiber length) leads to better compression, a reduced number of sidebands, and a higher power concentration in the pulse.

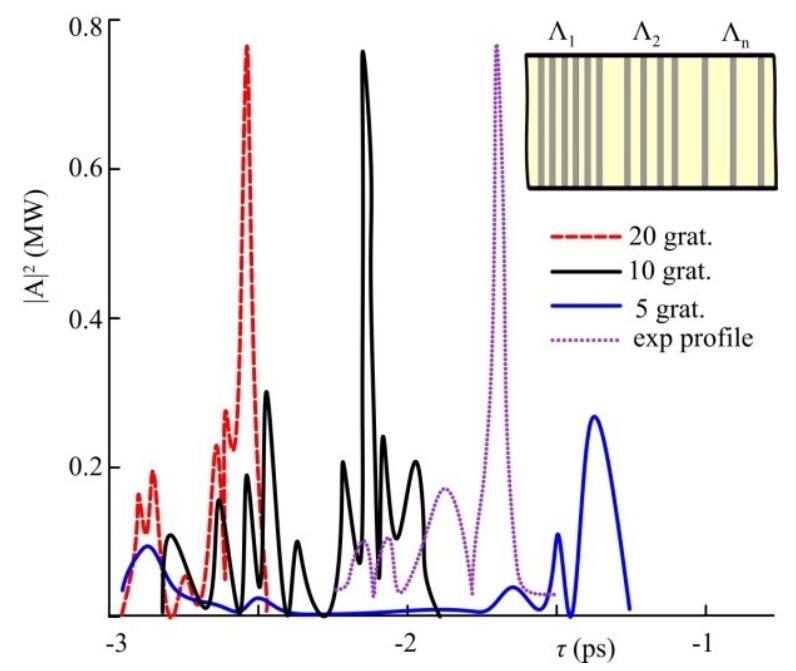

Figure 2. Temporal compression of frequency-modulated pulse in the passive fiber with inscribed FBGs providing the dispersion decreasing distribution along the fiber length. The initial pulse power is $7700 \mathrm{~W}$, the duration is $10 \mathrm{ps}$, and the fiber length is $20 \mathrm{~cm}$.

The use of passive fibers with inscribed FBG arrays as a means of pulse compression is possible if the input FM pulse contains enough energy. Therefore, we propose to use it as the second segment of the cascaded fiber configuration employing the active amplifying fiber as the first segment. The active fiber has to amplify the input pulse up to the required level. In the next section, we consider the cascaded fiber configuration comprising active and passive optical fibers with inscribed FBGs.

On the other hand, the type I-IR gratings allow a smooth variation of the grating period. For example, chirped gratings can be manufactured as the type I gratings. Thus, the choice of the optimal grating type for the proposed configuration is a subject to be studied separately elsewhere. However, we believe that both types can ensure significant results.

In our configuration, the modulation depth $m$ of the FBGs plays a crucial role. The greater the modulation depth, the shorter the FBG that can be used, and the higher GVD $\beta_{2}$ that can be achieved within a single FBG. As follows from relation (4), $\beta_{2}(z)$ increases as the square of the FBG modulation depth. According to [25,45], type II gratings inscribed with the pulsed laser are preferable for obtaining deeply modulated FBGs providing $m \gg 10^{-5}$.

On the other hand, the type I gratings allow a smooth variation of the grating period. For example, chirped gratings can be manufactured as type II gratings. Thus, the choice of the optimal grating type for the proposed configuration is a subject to be studied and published elsewhere. However, we believe that both FBG types are applicable for our purposes.

We consider the proposed configuration to be quite universal. Such FBGs can be effectively used in ytterbium-doped active fibers able to provide the generated pulses with high energy (consequently, high peak power over $100 \mathrm{~kW}$ ), and our configuration is primarily proposed to generate high power pulses. The simplest implementation of such technique can be considered using Ge-doped fibers [31,36], as the inscription technique is well developed for them [31]. Additionally, Er-doped active fibers are suitable for this purpose. 


\section{Amplification and Temporal Compression of FM Pulses in a Cascade of Active and Passive Fibers}

This section may be divided into subsections. It provides a concise and precise description of the experimental results, their interpretation, and the experimental conclusions that can be drawn.

The cascaded configuration comprising active and passive fibers of the same length $L$ with inscribed FBGs is presented in Figure $3 a$. Figure $3 b$ shows the distribution of the FBG period $\Lambda_{i}$ along the fiber length. A gradual decrease of the FBG period to a certain minimal value corresponds to the GVD increase.

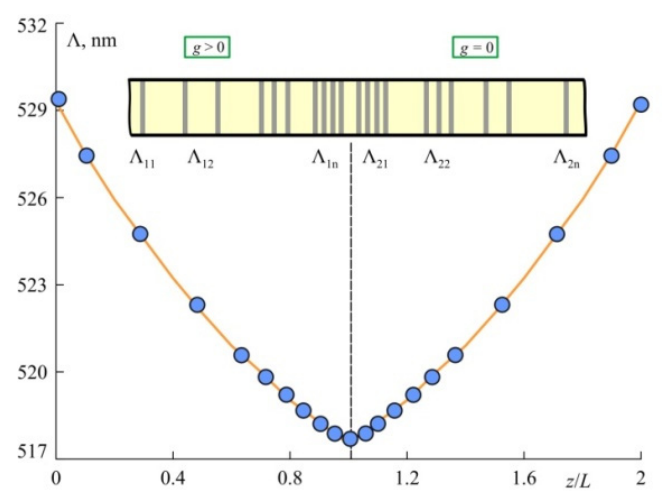

(a)

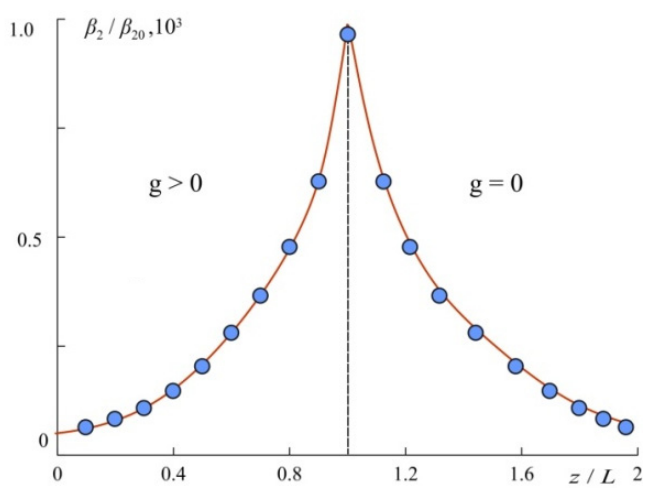

(b)

Figure 3. Arrangement of the inscribed FBGs (a) and the second-order dispersion profile (b) in the cascaded fiber configuration comprising one active and one passive fiber. The length of the active and passive fiber segment is $l=22.5 \mathrm{~cm}$, $g_{1}=0.2 \mathrm{~cm}^{-1}$, and $g_{2}=0$.

In the cascaded fiber configuration, the first fiber has a constant uniform gain $g_{1}>0$ along the whole fiber length, whereas in the second passive fiber, $g_{2}=0$. In the first fiber, the inscribed FBG period smoothly decreases, whereas in the second segment, it smoothly increases, achieving the initial level. The period of each FBG is set so that the GVD profile shall be approximated by the exponential functions $\beta_{2}=\beta_{20} \exp ( \pm q z)$ (Figure 3). In the first fiber, the FBG period decreases from 517.5 to $529.2 \mathrm{~nm}$, and the GVD increases by the factor $\beta_{2} / \beta_{20} \approx 10^{3}$. In the second fiber, the GVD decreases exponentially to its original value $\beta_{20}$.

Figure 4 shows the shape (a) and evolution (b) of the pulse as it propagates in the fiber cascade. In the active fiber, the input pulse experiences rather weak pulse temporal compression, and its power (black curve) increases up to $P \approx 16 \mathrm{~kW}$ (Figure 4 , red curve). In the passive fiber, a strong temporal compression with a drastic increase of the peak power up to a megawatt level occurs (Figure 4, blue curve).

Importantly, the pulse FM at the cascade output is nearly linear (Figure 4, inset). Therefore, the pulse is suitable for further temporal compression by standard tools, such as a pair of diffraction gratings. 

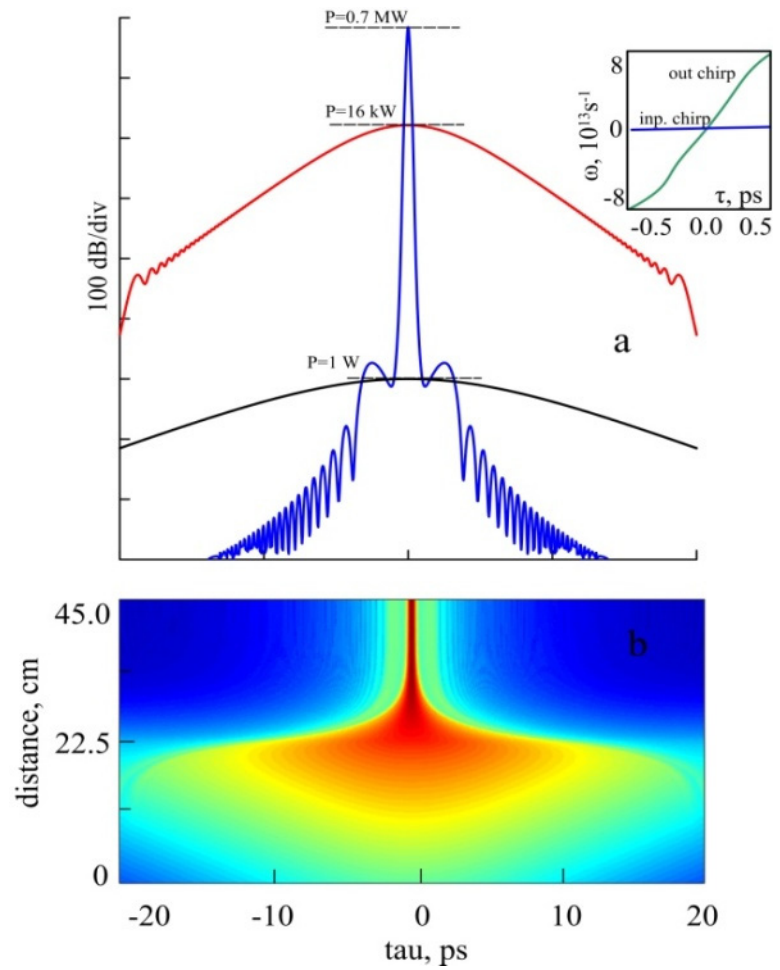

Figure 4. The shape (a) and evolution (b) of the pulse as it propagates in the fiber cascade. Temporal compression of FM pulse in the cascaded fiber configuration comprising one active and one passive fiber. The parameters, duration $\tau_{0}=10 \mathrm{ps}$, initial power $P_{0}=1 \mathrm{~W}$, initial frequency modulation $\alpha_{0}=10^{24} \mathrm{~s}^{-2}$, nonlinearity $R=10^{-5}(\mathrm{~W} \times \mathrm{m})^{-1}$, and dispersion profile are shown in Figure 3 .

\section{Generation of High Repetition Rate Trains of Soliton-Like Pulses}

In the previous section, we showed that the fiber cascaded configuration comprising one active and one passive optical fiber with inscribed FBGs enables a strong temporal compression and amplification of the chirped pulse. These mechanisms could be implemented at laser pulse parameters close to the parameters of FM solitons.

In fact, the proposed fiber configuration is also applicable for the generation of highrepetition rate soliton-like pulse (SLP) trains of high peak power from weakly modulated $\mathrm{CW}$ radiation. To achieve the effect, the cascaded fiber configuration under consideration should be spliced with the dispersion decreasing fiber (DDF) enabling the conversion of weakly modulated CW light into a train of low-power ultrashort pulses with a linear chirp.

The proposed fiber system is shown in Figure 5. Figure 6 presents the results of numerical simulation based on Equation (6) by the split-step Fourier method (SSFM) $[15,16]$.

A weakly modulated low-power optical signal introduced into the fiber configuration is expressed as:

$$
A(0, \tau)=\sqrt{P_{0}}\left[1+\chi \cos \left(\Omega_{\bmod } \tau\right)\right],
$$

where $\chi \ll 1$ is the signal modulation depth, $\Omega_{\bmod }=\omega_{0}-\omega_{v}$ is the modulation frequency, $\omega_{0}$ is the carrier frequency of the quasi-continuous optical signal, and $\omega_{v}$ is the frequency of the perturbation signal. The input signal power is $P_{0}=1 \mathrm{~W}$, and the modulation depth and frequency are $\chi=10^{-4}$ and $\Omega_{\bmod }=10^{12} \mathrm{~s}^{-1}$, respectively. 


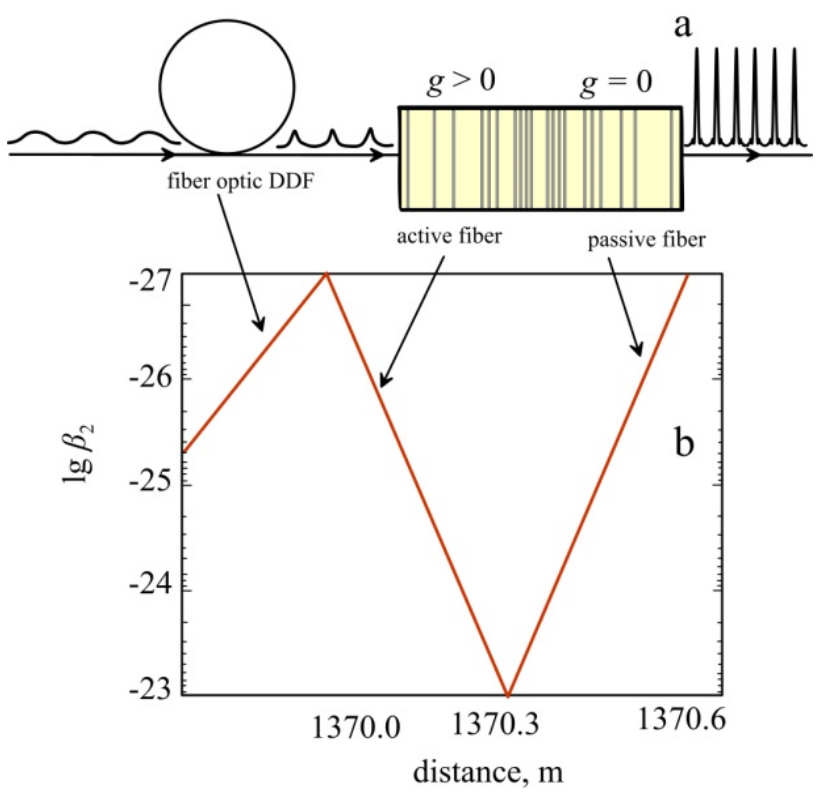

Figure 5. Fiber configuration comprising the DDF optical fiber and the cascade of active and passive fibers with inscribed FBG arrays (a). The GVD distribution over the fiber configuration length (b).

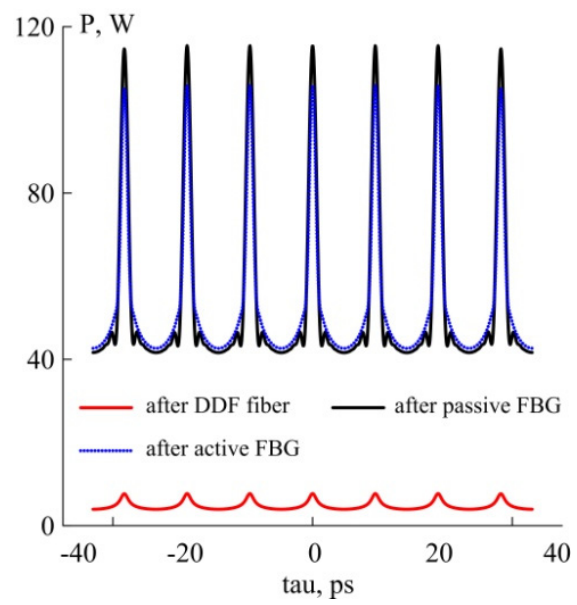

Figure 6. Generation of FM pulse trains in the cascade of active and passive fibers with the inscribed FBG arrays.

The parameters of the fibers used in the numerical simulation are listed in Table 1.

Table 1. Parameters of the fiber cascade elements.

\begin{tabular}{cccccc}
\hline $\begin{array}{c}\text { Cascade } \\
\text { Element }\end{array}$ & $\begin{array}{c}\text { Length } \\
\boldsymbol{l , \mathbf { m }}\end{array}$ & $\begin{array}{c}\text { Nonlinearity } \\
\boldsymbol{R},(\mathbf{W} \times \mathbf{m})^{-1}\end{array}$ & $\begin{array}{c}\text { Dispersion } \\
\boldsymbol{\beta}_{20}, \mathbf{s}^{2} / \mathbf{m}\end{array}$ & $\begin{array}{c}\text { Dispersion } \\
\boldsymbol{\beta}_{2}, \mathbf{s}^{2} \mathbf{m}\end{array}$ & $\begin{array}{c}\text { Gain } \\
\boldsymbol{g}_{0}, \mathbf{m}^{-1}\end{array}$ \\
\hline DDF fiber & 1370 & 0.001 & $-5 \times 10^{-26}$ & $-1 \times 10^{-27}$ & 0 \\
FBG fiber 1 & 0.3 & 0.001 & $-1 \times 10^{-27}$ & $-1 \times 10^{-23}$ & 6 \\
FBG fiber 2 & 0.3 & 0.001 & $-1 \times 10^{-23}$ & $-1 \times 10^{-27}$ & 0 \\
\hline
\end{tabular}

The evolution of the weakly modulated signal propagating in the cascade is as follows. In the DDF, because of modulation instability, the weakly modulated signal is converted into a train of low-power FM pulses (Figure 6, red curve) [15,16,43,44]. Then, in the amplifying optical fiber with the inscribed FBG array, the average power of the generated pulse train increases (adiabatic amplification), and the pulses are slightly compressed (blue curve). In the last stage, in the decreasing GVD passive optical fiber, the pulse receives its final temporal compression, resulting in the maximal output pulse peak power (black 
curve). In this way, the train of short picosecond pulses with a peak power of more than two orders of magnitude higher than the power of initial weakly modulated light is generated. It is worth noting that further optimization of the considered system is still possible. In particular, we observed that the use of $0.3 \mathrm{~m}$ longer passive fiber segments and better matching of the DDF fiber parameters enabled the peak power increases of up to $\sim 1 \mathrm{~kW}$.

\section{Conclusions}

We explored the evolution of FM pulses in fiber cascaded configurations comprising one active and one passive optical fiber with inscribed FBG arrays. It was shown that the use of chirped gratings with a "smooth" change in the period was most effective. In addition, we considered a technologically simpler version, which offers the use of a set of "uniform" gratings of finite length with different periods. This sequence of refractive index gratings is also capable of providing effective amplification, modulation, and compression of laser pulses up to sub-megawatt peak powers.

Both amplification and temporal compression in such a fiber cascade resulting in peak powers up to $\sim 700 \mathrm{~kW}$ were theoretically described. The quality of the generated pulse increases with the number of FBGs inscribed in the fiber of a given length. We showed that the amplified pulses could possess a linear frequency chirp, making them available for further compression. We also demonstrated the ability of the considered fiber cascades in conjugation with DDF optical fibers to generate terahertz picosecond pulse trains from a weakly modulated CW light. The peak power of the pulses in the train achieved a sub-kW level. Higher peak power pulses are probably available with systems comprising several cascades similar to those considered in the work.

Author Contributions: Conceptualization, I.Z. and A.F.; methodology, V.K. and A.A.; software, A.A., M.Y. and V.P.; validation, V.K., V.P., D.S. and A.T.; formal analysis, I.Z.; investigation, A.A. and A.F.; resources, I.Z. and A.F.; data curation, A.A.; writing-original draft preparation, A.F.; writing-review and editing, M.Y.; visualization, A.A.; supervision, I.Z.; project administration, I.Z. and A.F.; funding acquisition, A.F. All authors have read and agreed to the published version of the manuscript.

Funding: This research was funded by THE MINISTRY OF SCIENCE AND HIGHER EDUCATION OF THE RUSSIAN FEDERATION (MEGA-GRANT PROGRAM), grant number 075-15-2021-581 and the RUSSIAN FOUNDATION FOR BASIC RESEARCH, grant number 18-29-19101mk. A.A. thanks THE RF PRESIDENT FELLOWSHIP, fellowship number SP-4058.2021.5. The work of I.Z. and A.F. was supported by THE RUSSIAN SCIENCE FOUNDATION, grant number 18-12-00457P.

Institutional Review Board Statement: Not applicable.

Informed Consent Statement: Not applicable.

Data Availability Statement: Not applicable.

Conflicts of Interest: The authors declare no conflict of interest.

\section{References}

1. Geddes, C.G.R.; Toth, C.; Van Tilborg, J.J.; Esarey, E.; Schroeder, C.; Bruhwiler, D.; Nieter, C.; Cary, J.; Leemans, W.P. High-quality electron beams from a laser wakefield accelerator using plasma-channel guiding. Nat. Cell Biol. 2004, 431, 538-541. [CrossRef]

2. Veisz, L.; Schmid, K.; Tavella, F.; Benavides, S.; Tautz, R.; Herrmann, D.; Buck, A.; Hidding, B.; Marcinkevičius, A.; Schramm, U.; et al. Laser-driven electron acceleration in plasmas with few-cycle pulses. Comptes. Rendus. Phys. 2009, 10, 140-147. [CrossRef]

3. Buck, A.; Wenz, J.; Xu, J.; Khrennikov, K.; Schmid, K.; Heigoldt, M.; Mikhailova, J.M.; Geissler, M.; Shen, B.; Krausz, F.; et al. Shock-Front Injector for High-Quality Laser-Plasma Acceleration. Phys. Rev. Lett. 2013, 110, 185006. [CrossRef] [PubMed]

4. Caldwell, A.; Lotov, K.; Pukhov, A.; Simon, F. Proton-driven plasma-wakefield acceleration. Nat. Phys. 2009, 5, 363-367. [CrossRef]

5. Renninger, W.H.; Wise, F.W. Optical solitons in graded-index multimode fibres. Nat. Commun. 2013, 4, 1719. [CrossRef] [PubMed]

6. Krupa, K.; Tonello, A.; Barthélémy, A.; Couderc, V.; Shalaby, B.M.; Bendahmane, A.; Millot, G.; Wabnitz, S. Observation of geometric parametric instability induced by the periodic spatial self-imaging of multimode waves. Phys. Rev. Lett. 2016, 116, 183901. [CrossRef] [PubMed]

7. Krupa, K.; Tonello, A.; Shalaby, B.M.; Fabert, M.; Barthélémy, A.; Millot, G.; Wabnitz, S.; Couderc, V. Spatial beam self-cleaning in multimode fibres. Nat. Photonics 2017, 11, 237. [CrossRef] 
8. Ahsan, A.S.; Agrawal, G.P. Graded-index solitons in multimode fibers. Opt. Lett. 2018, 43, 3345-3348. [CrossRef] [PubMed]

9. Agrawal, G.P. Invite paper: Self-imaging in multimode graded-index fibers and its impact on the nonlinear phenomena. Opt. Fiber Technol. 2019, 50, 309-316. [CrossRef]

10. Filippov, V.; Chamorovskii, Y.; Kerttula, J.; Golant, K.; Pessa, M.; Okhotnikov, O.G. Double clad tapered fiber for high power applications. Opt. Express 2008, 16, 1929-1944. [CrossRef]

11. Trikshev, A.I.; Kurkov, A.S.; Tsvetkov, V.B.; Filatova, S.A.; Kertulla, J.; Filippov, V.; Chamorovskiy, Y.K.; Okhotnikov, O.G. A 160 W single-frequency laser based on an active tapered double-clad fiber amplifier. Laser Phys. Lett. 2013, 10, 065101. [CrossRef]

12. Andrianov, A.V.; Koptev, M.Y.; Anashkina, E.; Murav'Ev, S.V.; Kim, A.V.; Lipatov, D.S.; Velmiskin, V.; Levchenko, A.E.; Bubnov, M.M.; Likhachev, M.E. Tapered erbium-doped fibre laser system delivering $10 \mathrm{MW}$ of peak power. Quantum Electron. 2019, 49, 1093-1099. [CrossRef]

13. Kuznetsov, A.G.; Kharenko, D.S.; Babin, S.A. Amplification of dissipative solitons with a polarisation-maintaining tapered fibre amplifier. Quantum Electron. 2018, 48, 1105-1108. [CrossRef]

14. Dong, L.; Peng, X.; Li, J. Leakage channel optical fibers with large effective area. J. Opt. Soc. Am. B 2007, 24, 1689-1697. [CrossRef]

15. Kivshar, Y.S.; Agrawal, G.P. Optical Solitons: From Fibers to Photonic Crystals; Academic Press: New York, NY, USA, 2003 ; p. 125.

16. Agrawal, G.P. Nonlinear Fiber Optics, 4th ed.; Springer: New York, NY, USA, 2007; p. 530.

17. Litchinitser, N.; Eggleton, B.; Patterson, D. Fiber Bragg gratings for dispersion compensation in transmission: Theoretical model and design criteria for nearly ideal pulse recompression. J. Light. Technol. 1997, 15, 1303-1313. [CrossRef]

18. Lenz, G.; Eggleton, B.J. Adiabatic Bragg soliton compression in nonuniform grating structures. J. Opt. Soc. Am. B 1998, 15, 2979-2985. [CrossRef]

19. Lenz, G.; Eggleton, B.J.; Litchinitser, N. Pulse compression using fiber gratings as highly dispersive nonlinear elements. J. Opt. Soc. Am. B 1998, 15, 715-721. [CrossRef]

20. Qian, L.; Senthilnathan, K.; Nakkeeran, K.; Wai, P.K.A. Nearly chirp and pedestal free pulse compression in nonlinear fiber Bragg gratings. J. Opt. Soc. Am. B 2009, 26, 432-443.

21. Qui, L.; Wai, P.K.A.; Senthilnathan, K.; Nakkeeran, K. Modeling self-similar optical pulse compression in nonlinearfiber bragg grating using coupled mode equation. J. Lightwave Technol. 2011, 29, 1293-1305.

22. Vasil'Ev, S.A.; Medvedkov, O.I.; Korolev, I.G.; Bozhkov, A.S.; Kurkov, A.S.; Dianov, E.M. Fibre gratings and their applications. Quantum Electron. 2005, 35, 1085-1103. [CrossRef]

23. Kablukov, S.I.; Zlobina, E.A.; Skvortsov, M.I.; Nemov, I.N.; Wolf, A.; Dostovalov, A.V.; Babin, S.A. Mode selection in a directly diode-pumped Raman fibre laser using FBGs in a graded-index multimode fibre. Quantum Electron. 2016, 46, 1106-1109. [CrossRef]

24. Zlobina, E.A.; Kablukov, S.; Wolf, A.; Dostovalov, A.V.; Babin, S.A. Nearly single-mode Raman lasing at $954 \mathrm{~nm}$ in a graded-index fiber directly pumped by a multimode laser diode. Opt. Lett. 2016, 42, 9-12. [CrossRef] [PubMed]

25. Zlobina, E.A.; Kablukov, S.; Skvortsov, M.I.; Nemov, I.N.; Babin, S.A. $954 \mathrm{~nm}$ Raman fiber laser with multimode laser diode pumping. Laser Phys. Lett. 2016, 13, 035102. [CrossRef]

26. Wolf, A.A.; Dostovalov, A.V.; Wabnitz, S.; Babin, S.A. Femtosecond writing of refractive index structures in multimode and multicore optical fibres. Quantum Electron. 2018, 48, 1128-1131. [CrossRef]

27. Wolf, A.; Dostovalov, A.; Bronnikov, K.; Babin, S. Arrays of fiber Bragg gratings selectively inscribed in different cores of 7-core spun optical fiber by IR femtosecond laser pulses. Opt. Express 2019, 27, 13978-13990. [CrossRef]

28. Mizunami, T.; Djambova, T.; Niiho, T.; Gupta, S. Bragg gratings in multimode and few-mode optical fibers. J. Light. Technol. 2000, 18, 230-235. [CrossRef]

29. Sang, X.; Yu, C.; Yan, B. Bragg gratings in multimode optical fibers and their applications. J. Optoelectron. Adv. Mater. 2006, 8, 1616.

30. Liu, Y.; Lit, J.; Gu, X.; Wei, L. Fiber comb filters based on UV-writing Bragg gratings in graded-index multimode fibers. Opt. Express 2005, 13, 8508-8513. [CrossRef]

31. Kashyap, R. Fiber Bragg Gratings; Academic Press: Cambridge, MA, USA, 1999.

32. Kurkov, A.S.; Grukh, D.A.; Medvedkov, O.I.; Paramonov, V.M.; Dianov, E.M.; Yashkov, M.V.; Vechkanov, N.I.; Guryanov, A.N. Multimode fiber lasers based on Bragg gratings and double-clad Yb-doped fibers. Laser Phys. Lett. 2004, 1, 473-475. [CrossRef]

33. Jha, R.; Villatoro, J.; Badenes, G. Ultrastable in reflection photonic crystal fiber modal interferometerfor accurate refractive index sensing. Appl. Phys. Lett. 2008, 93, 191106. [CrossRef]

34. Okawara, C.; Saijyou, K. Fiber optic interferometric hydrophone using fiber Bragg grating with wavelength division multiplexing. Acoust. Sci. Technol. 2008, 29, 232-234. [CrossRef]

35. Cusano, A.; Cutolo, A.; Albert, J. (Eds.) Fiber Bragg Grating Sensors: Recent Advancements. In Industrial Applications and Market Exploitation; Bentham Science Publishers: Beijing, China, 2011.

36. Medvedkov, O.I.; Vasiliev, S.A.; Gnusin, P.I.; Dianov, E.M. Photosensitivity of optical fibers with extremely high germanium concentration. Opt. Mater. Express 2012, 2, 1478-1489. [CrossRef]

37. Leal-Juniora, A.G.; Díaza, C.A.R.; Frizeraa, A.C.; Anselmo, F.; Marquesb, C.; Ribeiroa, M.R.N.; José Pontes, M. Simultaneous measurement of pressure and temperature with a single FBG embedded in a polymer diaphragm. Opt. Laser Technol. 2019, 112, 77-84. [CrossRef]

38. Allsop, T.; Mou, C.; Neal, R.; Kundrát, V.; Wang, C.; Kalli, K.; Webb, D.; Liu, X.; Davey, P.; Culverhouse, P.; et al. Generation of a Conjoint Surface Plasmon by an Infrared Nano-Antenna Array. Adv. Photonics Res. 2021, 2, 2000003. [CrossRef] 
39. Eggleton, B.J.; Slusher, R.E.; de Sterke, C.M.; Krug, P.A.; Sipe, J.E. Bragg Grating Solitons. Phys. Rev. Lett. 1996, 76, 1627-1630. [CrossRef] [PubMed]

40. Eggleton, B.; de Sterke, C.; Aceves, A.; Sipe, J.; Strasser, T.; Slusher, R. Modulational instability and tunable multiple soliton generation in apodized fiber gratings. Opt. Commun. 1998, 149, 267-271. [CrossRef]

41. Serkin, V.N.; Hasegawa, A. Novel Soliton Solutions of the Nonlinear Schrödinger Equation Model. Phys. Rev. Lett. 2000, 85, 4502-4505. [CrossRef]

42. Melchor, G.M.; Granados, M.A.; Corro, G.H. On the problem of ideal amplification of optical solitons. Quantum Electron. 2002, 32, 1020-1028. [CrossRef]

43. Korobko, D.A.; Okhotnikov, O.G.; Zolotovskii, I.O. High-repetition-rate pulse generation and compression in dispersion decreasing fibers. JOSA B 2013, 30, 2377-2386. [CrossRef]

44. Korobko, D.A.; Okhotnikov, O.G.; Stoliarov, D.A.; Sysoliatin, A.A.; Zolotovskii, I.O. Highly Nonlinear Dispersion Increasing Fiber for Femtosecond Pulse Generation. J. Light. Technol. 2015, 33, 3643-3648. [CrossRef]

45. Dostovalov, A.; Wolf, A.; Parygin, A.; Zyubin, V.; Babin, S. Femtosecond point-by-point inscription of Bragg gratings by drawing a coated fiber through ferrule. Opt. Express 2016, 24, 16232-16237. [CrossRef] [PubMed] 